\title{
Energy up conversion caused by metallic photonic boxes
}

\author{
Wo-Chung Liu, Cha-Hsin Chao, Chia-Wei Tsai, Ching-Fuh Lin* \\ Graduate Institute of Electro-Optical Engineering, National Taiwan University, Taipei, Taiwan, ROC \\ *also with Graduate Institute of Electronics Engineering and Department of Electrical Engineering
}

\begin{abstract}
A photonic box structure is discovered to have the characteristic of transferring part of the input pumping $532 \mathrm{~nm}$ laser energy to wavelength $300 \mathrm{~nm}$. The spectral width is narrower and the intensity is linearly increased as the intensity of the input laser increases. The spectral width shows an abrupt change when input laser power exceeds $30 \mathrm{~mW}$. The mechanism of this phenomenon is considered to be related to surface plasmon resonance, however, complete explanation cannot be obtained through known physics. This new energy transfer phenomenon gives us a new direction toward using the photonic box structure.
\end{abstract}

Index Terms - photonic crystal, wavelength conversion, energy transfer.

\section{INTRODUCTION}

In spite of great advance of laser sources in the past decades, some wavelength bands are still not available, hindering applications of lasers from some areas. Because finding suitable laser materials for all the wavelengths cannot be achieved, wavelength conversion is highly desired. [1]

Conventional wavelength conversion includes following methods. The first type is the four wave mixing (FMW), which is a type of optical Kerr effect occurring when light of two or more different wavelengths is launched into a fiber or other media with the Kerr effect. Generally speaking, FWM occurs when light of three different wavelengths is launched into a fiber, giving rise to a new wave (known as an idler), the wavelength of which does not coincide with any of the others [2]. The second type is the Raman scattering [3], which can be used to change the laser emission wavelength over a fixed frequency shift, making access to additional spectral output of laser lines. The spectral areas reachable with Raman scattering extend from ultraviolet to near infrared, depending on the pump laser and Raman material used. If the Raman medium is pumped hard enough, stimulated Raman scattering (SRS) processes can also occur, giving rise to very efficient wavelength conversion. Efficient SRS has been experimentally realized in liquids, gases, and also in solid state materials [4][5]. SRS can therefore be used as a supplement or an alternative to the nonlinear techniques [6][7]. The third type is the optical nonlinearity, which can be used for second harmonic generation(SHG) [8] and parametric oscillation/amplification [9]. With this type of nonlinear optics, the wavelength of the pumping beam can be doubled or down-shifted.

Recently, nonlinear frequency conversion in 2D photonic crystals is also theoretically and experimentally studied. Such structures can possibly be used for applications as multiple-beam second-harmonic generation (SHG), ring cavity SHG, or multiple wavelength frequency conversion [10]. In addition, photonic crystal fibers have been discovered to generate very broadband wavelength conversion [11].

Here we report a new way of wavelength conversion using photonic boxes. Photonic boxes have been found to be able to modify the black-body radiation for enhancing visible spectrum [12]. The term "photonic box" is defined here as the metallic box that has the dimension in the order of the wavelength of light. In this work, we use 532nm laser as the pumping light. Photonic boxes are able to transform $532 \mathrm{~nm}$ laser energy to light with wavelength of $300 \mathrm{~nm}$. The up-converted energy is linearly increased with the intensity of the pumping laser. This up-conversion of laser energy is much more efficient than the usual Raman scattering. Wavelength conversion discovered in the past is often nonlinearly increased with the pumping [13]. The linear property of the energy up conversion in this work indicates that the mechanism here is novel. The detailed physics will be discussed.

\section{EXPERIMENT}

Cubic photonic box with the size of $250 \mathrm{~nm}$ is fabricated on silicon wafer. The fabrication procedures are as follows. First, metal W/Cr/Au/Pt with the thickness of $400 \mathrm{~nm}, 10 \mathrm{~nm}, 10 \mathrm{~nm}$, and $20 \mathrm{~nm}$ was sequentially evaporated on the Si substrate, respectively. Metals $\mathrm{Cr}$ and $\mathrm{Au}$ are used to enhance the adhesion of $\mathrm{Si}$ and $\mathrm{Pt}$. $\mathrm{SiO}_{2}$ of thickness $250 \mathrm{~nm}$ is then deposited using E-gun evaporator at the surface of Pt. Afterwards, photoresist (PR) was spun on $\mathrm{SiO}_{2}$ for photolithography. IIPL 
(Immersion interference photolithography) using a 351 $\mathrm{nm}$ Argon ion laser was applied twice to create two dimensional periodic patterns on the photoresist, which forms square pattern of $250 \mathrm{~nm}$ length and $250 \mathrm{~nm}$ spacing. This pattern was later transferred to the $\mathrm{SiO}_{2}$ under it by using reactive-ion-etching (RIE) process. During RIE, Ar and $\mathrm{CHF}_{3}$ were used to dry etch the $\mathrm{SiO}_{2}$ uncovered by PR. After the desired pattern of $\mathrm{SiO}_{2}$ was formed, PR above it was rinsed off by acetone. The device fabrication was completed by evaporating $250 \mathrm{~nm} \mathrm{Pt}$ again to fill in the spaces between $\mathrm{SiO}_{2}$ cubes. A cross section view of the device is available through scanning electron microscope image and was shown on Fig. 1. The length and height of $\mathrm{SiO}_{2}$ is found to be $250 \mathrm{~nm}$ and $350 \mathrm{~nm}$, respectively.

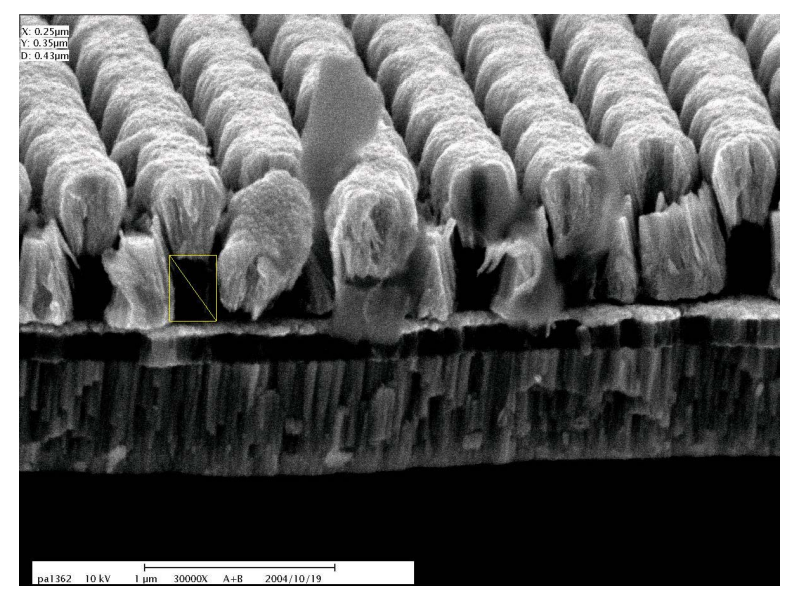

Fig. 1 SEM cross section view of the metallic photonic box sample structure. The length and height of $\mathrm{SiO} 2$ is $250 \mathrm{~nm}$ and $350 \mathrm{~nm}$, respectively.

The experiment setup is similar to photoluminescence measurement. The pumping source used here is $532 \mathrm{~nm}$ green light laser. Pumping source emitted by the laser first passes through a neutral density filter, which is used to adjust the output power of the laser. A power meter is placed in the light path between the sample and the neutral density filter, the desired laser output power is obtained by reading the power meter and rotating the neutral density filter. A lens is placed between the green laser and sample to focus the green laser energy to a small spot with about $0.5 \mathrm{~mm}$ diameter. After the green laser emitted to the sample, two lenses are used to collect the scattered light emitted from the sample to the monochromator. A detector attached behind the monochromator is used to measure the light intensity at different wavelengths. These data are then recorded into a computer.

A light emitted at wavelength $300 \mathrm{~nm}$ is measured. If the pumping power is increased gradually, as shown in Fig. 2, an intensity increase is observed. This suggests that the structure of our sample is capable of absorbing the laser energy at $532 \mathrm{~nm}$ and then transform part of the energy into wavelength $300 \mathrm{~nm}$. Peak intensity and spectral area linearly increases with a constant slope according to pumping power (Fig. 3). The most interesting part lies in that the spectral width becomes narrower as the laser input power increased, which can be observed in Fig 4. The output peak wavelength remains constant at $300 \mathrm{~nm}$ as the pumping laser power increases, indicated by Fig5.

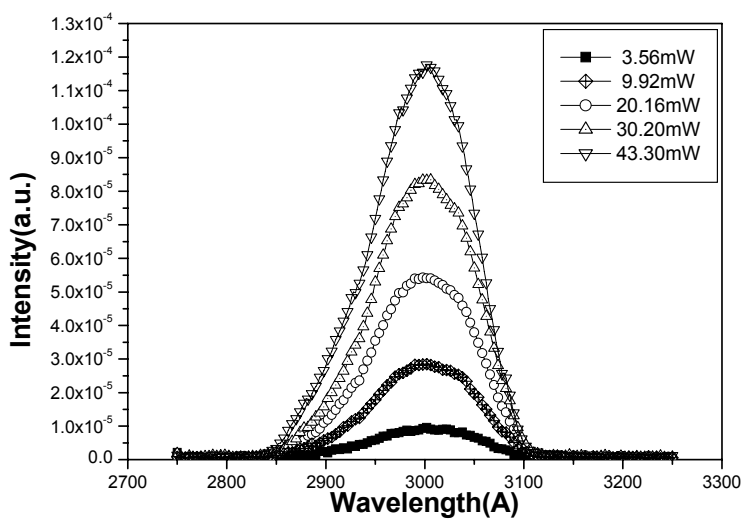

Fig. 2 Light emitted at wavelength $300 \mathrm{nn}$ shows a linear increase with the increase of input laser intensity.

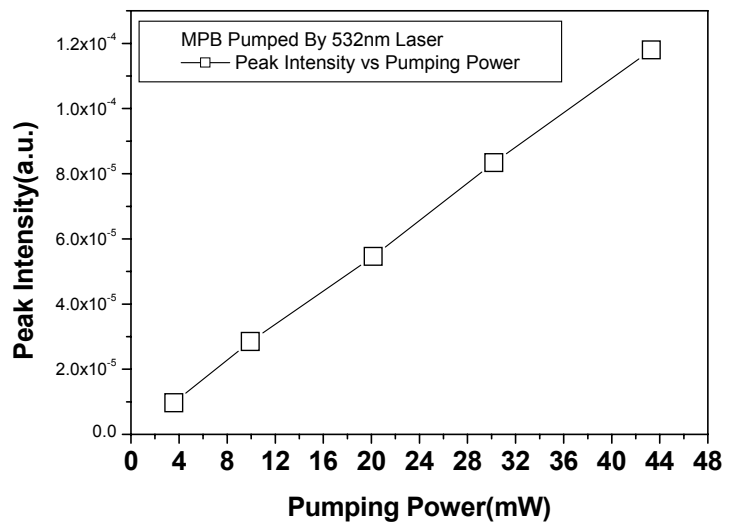

Fig. 3 Peak intensity of the light emitted by the structure shows constant slope increase versus pumping power. 


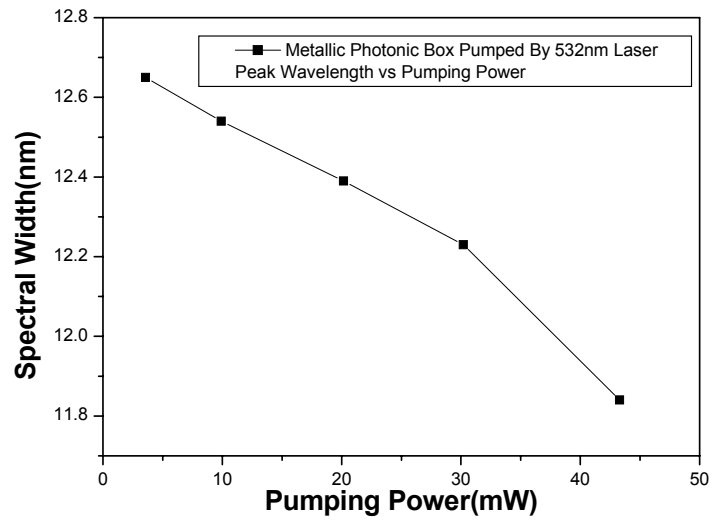

Fig. 4 Spectral width becomes narrower as the input pumping power increased. An abrupt slope change is observed when laser power exceeds $30 \mathrm{~mW}$..

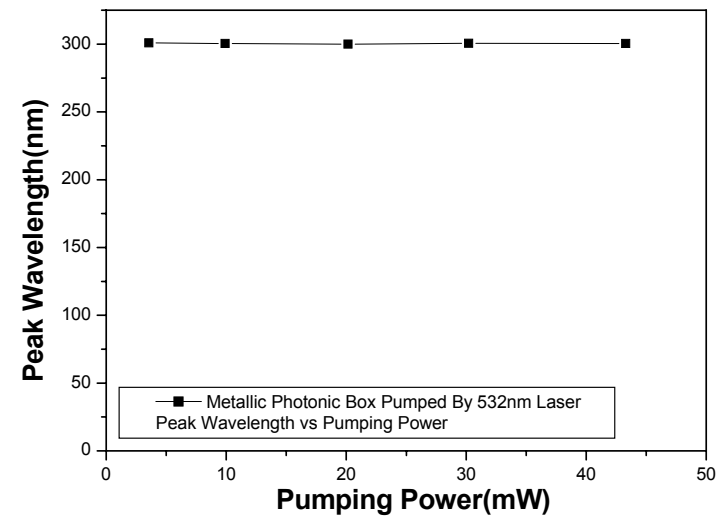

Fig. 5 peak wavelength remains constant as pumping laser power increases...

\section{DISCUSSION}

The energy transfer mechanism observed here may result from the following mechanisms: (1)Surface plasmon resonance, an electron charge density wave phenomenon that arises at the surface of a metallic film when light is reflected at the film under specific conditions. The resonance is a result of energy and momentum being transformed from incident photons into surface plasma, and is sensitive to the refractive index of the medium on the opposite side of the film from the reflected light. Here the structure we designed is dielectric medium enclosed by thin metal Pt. The laser energy was directly input to the nano-structure and causes energy of wavelength at $300 \mathrm{~nm}$ to be amplified through surface plasmon resonance. (2)Rectangular cavity resonator is defined as dielectric material enclosed by metal [14]. Due to the boundary condition of the electromagnetic waves at the conducting metal surface, only the electromagnetic waves with specific frequencies are allowed to propagate within this cavity. The resonant frequency is defined as follows:

$$
f_{k l m}=\frac{u}{2} \sqrt{\left(\frac{k}{a}\right)^{2}+\left(\frac{l}{b}\right)^{2}+\left(\frac{m}{d}\right)^{2}}
$$

Here $\mathrm{u}$ indicates the light velocity in the dielectric material. a, b and d stands for the length, width and height of the cavity. $\mathrm{k}, 1$ and $\mathrm{m}$ are integers and repreesnt the footnote of the TE/TM standing wave. The propagating mode with the lowest cut-off frequency is defined as the dominant mode. By using equation (1) and the relation between frequency and wavelength $\lambda=u / f$, one can easily calculate the longest cut-off wavelength of such cavity resonator. Electromagnetic waves with wavelength longer than cut-off wavelength are not allowed to propagate within the cavity resonator. By substituting a, b, d with $250 \mathrm{~nm}, 250 \mathrm{~nm}, 350 \mathrm{~nm}$, we can calculate the highest cut-off wavelength to be $610.3 \mathrm{~nm}$. The signal we measured is located at $300 \mathrm{~nm}$, which is about half of this wavelength. As the pumping laser is $532 \mathrm{~nm}$, it seems pretty reasonable that signal of $610.3 \mathrm{~nm}$ does not appear. However, this theory still cannot explain the narrowing of the spectral width and the absence of other signals with different propagating modes.

\section{CONCLUSION}

A metallic photonic box structure being able to convert part of the input energy of $532 \mathrm{~nm}$ laser to $300 \mathrm{~nm}$ is discovered. The converted energy intensity is linearly increased with the input laser power. We also find that the spectral width becomes narrower as the input laser energy increases, and the narrowing rate is about constant except for input laser power exceeds $30 \mathrm{~mW}$. The energy transfer phenomenon is considered to result from surface plasmon resonance or rectangular cavity resonator theory. Nevertheless, neither theory is able to perfectly explain the facts we observed. There may be new physics lie behind this experiment and the explanation still remains open.

This discovery is very exciting because it is believed that with the variation of the structure size, energy 
transformation at any desired wavelength can be easily obtained. Another possible application of this structure lies in the combination of organic light emitting diode(OLED). It is believed that $40 \%$ of the energy emitted by OLED will be converted to surface plamon and trapped in the thin metal layer [15]. The structure we proposed here is possible to employ this part of the energy and transfer the light to the wavelength desired.

\section{ACKNOWLEDGEMENT}

The authors would like to deeply appreciate the help of IIPL lithography from W. C. Cheng and Prof. L.A. Wang, Graduate Institute of Electro-Optical Engineering, National Taiwan University, Taipei, Taiwan, R.O.C.

\section{REFERENCES}

[1] F. Benabid, G. Bouwmans et al., "Ultrahigh Efficiency Laser Wavelength Conversion in a Gas-Filled Hollow Core Photonic Crystal Fiber by Pure Stimulated Rotational Raman Scattering in Molecular Hydrogen" Phys. Rev. Lett. Vol.93 Num.12 (2004).

[2] Osamu Aso, Masateru Tadakuma and Shu Namiki, "FourWave Mixing in Optical Fibers and Its Applications" Furukawa Review, No. 19. 2000.

[3] Hongxing Xu, Xue-Hua Wang, Martin P. Persson, and H. Q. $\mathrm{Xu}$, "Unified Treatment of Fluorescence and Raman Scattering Processes near Metal Surfaces" Phys. Rev. Lett. 93, 243002 (2004)

[4] W. Kaiser, M. Maier, In: T. Arrecchi, E.O. Schultz-Dubois (Eds.), Laser Handbook, Vol. 2, FN-Holland, Amsterdam, 1972, p. 1077.

[5] Y.-R. Shen, In: M. Cardona (Ed.), Light Scattering in Solids, Vol. 1, Springer, Berlin, 1983, p. 2

[6] Pavel Cerny, Helena Jelinkova, Peter G. Zverev and Tasoltan T. Basiev, "Solid state lasers with Raman frequency conversion” Progress in Quantum Electronics 28 (2004) 113-143.

[7] N. Bloembergen, Nonlinear Optics, W.A. Benjamin, Inc., New York, 1965, pp.177-196.

[8] S. Bergfeld and W. Daum, "Second-Harmonic Generation in Galfs: Experiment versus Theoretical Predictions of $\chi_{x y z}$ " Phys. Rev. Lett. 90, 036801 (2003)

[9] Jean-Jacques Zondy and Dmitri Kolker, "Dynamical Signatures of Self-Phase-Locking in a Triply Resonant Optical Parametric Oscillator" Phys. Rev. Lett. 93, 043902 (2004)

[10] V. Berger, “ Nonlinear Photonic Crystals” Physical Review Letters Vol. 81, No. 19, 9 November 1998.

[11] S. Y. Lin, J. G. Fleming, and I. El-Kady, "Highly efficient light emission at lambda $=1.5$ um by a three-dimensional tungsten photonic crystal" OPTICS LETTERS Vol. 28, No. 18, Sep. 15, 2003.

[12] Cha-Hsin Chao, Ching-Fuh Lin et al., "Using the Metallic Photonic Box to Modify Blackbody Radiation for Illumination Purpose" opt2003.
[13] J Trull, R Vilaseca and Jordi Martorell, "Quadratic nonlinear radiation within a 1D photonic crystal" J. Opt. B: Quantum Semiclass. Opt. 1 (1999) 307-314.

[14] David K. Cheng, Field and Wave Electromagnetics, 2nd edition, chap. 10 .

[15] Stephen Wedge and W. L. Barnes, "Surface plasmonpolariton mediated light emission through thin metal films" 9 August 2004 / Vol. 12, No. 16 / OPTICS EXPRESS 3673 